Original Research Article

\title{
Comparative study of efficacy and safety of omega 3 fatty acids and fenofibrate with background atorvastatin therapy in patients of atherogenic dyslipidaemia
}

\author{
Ankit Arora $^{1}$, Navyug Raj Singh ${ }^{1 *}$, Rajiv Sharma ${ }^{2}$
}

\begin{abstract}
${ }^{1}$ Department of Pharmacology, Government Medical College, Amritsar, Punjab, India ${ }^{2}$ Department of Internal Medicine, Lifecare Hospital, Amritsar, Punjab, India

Received: 23 March 2019

Revised: 03 April 2019

Accepted: 03 May 2019

*Correspondence to:

Dr. Navyug Raj Singh,

Email: navyug69@gmail.com

Copyright: (C) the author(s), publisher and licensee Medip Academy. This is an openaccess article distributed under the terms of the Creative Commons Attribution NonCommercial License, which permits unrestricted noncommercial use, distribution, and reproduction in any medium, provided the original work is properly cited.
\end{abstract}

\begin{abstract}
Background: Trials of atorvastatin combined either with fenofibrate or with omega-3 fatty acids (O3FA) have shown promising results in atherogenic dyslipidemia but there are very few studies where both these TGs lowering agents have been compared with each other. This study was conducted to compare efficacy and safety of these two agents on lipid profile of patients of atherogenic dyslipidaemia on background statin therapy and also to monitor effects of these interventions on serum uric acid (SUA) levels.

Methods: About 90 patients of dyslipidemia were randomised to 3 groups and received O3FA (2000 $\mathrm{mg})$, fenofibrate $(80 \mathrm{mg}$ ) or dietary restrictions, each with atorvastatin $(20 \mathrm{mg})$ in background for a period of 90 days. Total cholesterol (TC), HDL-C,TGs, LDL-C, SGOT and SGPT levels were done at baseline, 6 weeks and 12 weeks. Other parameters (SUA and BMI) were done at baseline and 12 weeks.

Results: Both group 1 (O3FA) and group 2 (fenofibrate) showed highly significant fall in TG levels $(\mathrm{p}<0.001)$ in comparison to group 3 (dietary restrictions) whereas comparative TG reduction between groups 1 and group 2 was not significant. Group 2 also showed significant fall in LDL-C levels ( $p$ $<0.01$ ) in comparison to group 3. LDL-C reduction, TG reduction and SUA reduction was more in group 2 compared to group 1 followed by group 3 . No significant difference was observed in the incidence of adverse effects in three study groups.

Conclusions: Combination of fenofibrate and atorvastatin was more effective than that of omega-3 fatty acid and atorvastatin, in lowering serum TG and LDL$\mathrm{C}$ levels. There was a significant reduction in SUA levels in all three groups, but combination of fenofibrate and atorvastatin again showed better outcomes. With respect to the safety, all the 3 groups were comparable. O3FA, however, may be a good alternative to fibrates in patients not tolerating latter.
\end{abstract}

Keywords: Atherogenic dyslipidaemia, Atorvastatin, Fenofibrate, Hypertriglyceridemia, Omega-3 fatty acids

\section{INTRODUCTION}

Dyslipidemia appears to be widely prevalent in India (20\% to $35 \%) .{ }^{1}$ More important is the pattern of dyslipidemia in Indians and migrant Asian Indians, tendency to have higher TGs and lower HDL-C levels due to their less physical activity and consumption of carbohydrate and fat rich diet. Besides, they have higher intra-abdominal visceral fat causing increased insulin resistance and CVD risk than westerners. ${ }^{2-5}$
Atherogenic dyslipidemia (AD) is a clinical condition characterized by elevated levels of triglycerides (TGs) and small-dense low-density lipoprotein (sdLDL) particle and low levels of high-density lipoprotein cholesterol (HDLC) and are recognized as independent risk factors for cardiovascular disease. ${ }^{6}$ There is also presence of elevated levels of VLDL, apolipoprotein B and oxidized LDL. ${ }^{7}$ It is often observed in patients with metabolic syndrome (MS), obesity, insulin resistance and type 2 diabetes mellitus, hence also referred as either diabetic dyslipidemia or 
dyslipidemia of metabolic syndrome and is considered as an important CVD (cardiovascular disease) risk factor in these patients. ${ }^{8,9}$ The another risk factor shown to be a predictor of stroke, coronary artery disease and metabolic syndrome is raised levels of serum uric acid. ${ }^{10}$ However, the definite role of uric acid in these diseases is still the subject of much discussion, because it is always accompanied with other risk factors such as diet, obesity, and dyslipidemia. ${ }^{11,12}$ It is reported that hypolipidaemic agents statins and fibrates may also reduce the SUA levels. ${ }^{13,14}$

In $\mathrm{AD}$, impaired insulin signalling causes increased lipolysis in adipocytes and culminates into overproduction of VLDL-1 in the liver which leads to increase in sdLDL particle production and decrease in HDL-C, substantially influencing the development of atherosclerosis. ${ }^{15-17}$ Increase in sdLDL generation has been noted when TG levels are $>133 \mathrm{mg} / \mathrm{dl} .{ }^{18}$ Oxidized LDL also plays a significant role in AD. It originates from LDL or sdLDL by mild oxidation in the arterial wall by cell-associated lipoxygenase and/or myeloperoxidase and cause endothelial dysfunction resulting in atherosclerosis. ${ }^{19,20}$

The metabolic disturbance begins with increased production of VLDL-TG and ends in atherogenic reduction of HDL-C, intravascular remodelling, and reduced reverse cholesterol transport from peripheral tissues, hepatocytes and macrophages to liver, further aggravating atherosclerosis. ${ }^{15}$ Raised TGs are attributed to high carbohydrates $(>60 \%)$ in diet, taken specially in the form of simple sugars. Raised sdLDL levels and decreased HDL levels are attributed to the higher dietary intake of SFAs.It was reported that overall carbohydrate restriction improves atherogenic lipid states even in absence of actual weight loss and also, that restricting dietary fats to $25-35 \%$ of calories with complex carbohydrates and high fibre intake facilitates TGs lowering, increments in HDL-C levels and produce large buoyant LDL particles (less atherogenic). Secondly, a significant improvement in HDL-C levels (4-22\%) may be seen with physical activity. Nutrition in all forms of dyslipidaemia management should be individualized. ${ }^{1}$ Avoidance of alcohol intake and smoking cessation must be undertaken. This can be supplemented with drug therapies such as statin monotherapy or combination therapy with niacin/fibrates. ${ }^{15}$

Statins are most effective in reducing LDL-C level and raising HDL-C level as well. Combination with other drug classes helps in reducing triglyceride level. It is suggested that when plasma triglycerides exceed a critical threshold of approximately $133 \mathrm{mg} / \mathrm{dl}(1.5 \mathrm{mmol} / \mathrm{l})$, this favors the formation of small, dense LDL from larger, less dense species. Statins are capable of lowering triglyceride levels below this threshold value will cause a shift to a less dense and, therefore, less atherogenic LDL profile. ${ }^{18}$ Fibrates reduce triglycerides through PPAR $\alpha$-mediated stimulation of fatty acid oxidation, increased LPL synthesis, and reduced expression of apo C-III. An increase in LPL would enhance the clearance of triglyceride-rich lipoproteins. Fibrate mediated increases in HDL-C are due to PPAR $\alpha$ stimulation of apo A-I and apo A-II expression, which increases HDL levels. Combination of fibrates with statin may be required in the management of hypertriglyceridemia and atherogenic dyslipidemia for better control but there are major safety issues including higher chances of myopathy, rhabdomyolysis and nephrotoxicity. ${ }^{21}$ But fenofibrate has less interaction with statins and can be used safely with atorvastatin.

O3FA: DHA (Docosahexaenoic acid), EPA (Eicosapentaenoic acid) and Alphalinolenic acid (ALA) are PUFAs which are useful in treating hypertriglyceridemia. They reduce VLDL production by up-regulation of LDL receptors and thus increasing catabolic rate of LDL-C and also inhibit esterification of other fatty acids, while they themselves are inefficient substrates for enzymes involved in triglyceride synthesis. Also, inhibition of acylCoA cholesterol acyl transferase (ACAT) and increased hepatic peroxisomal $\beta$ oxidation resulting in reduced cholesterol synthesis and resulting in increased clearance of fatty acids, respectively. Ultimately, they reduce the atherogenic tendencies as compared to saturated fatty acid. ${ }^{22}$ The combination therapy of O3FA and statins have shown significant (20\%$30 \%$ ) reduction in triglycerides and significant (30\%-40\%) reduction in VLDL level as compared to statin alone. ${ }^{23}$

Therefore, in combined dyslipidemia, combination of statins with other lipid lowering agents (which decrease triglyceride levels like fibrates, niacin or O3FAs) is more effective.

Present study compares primarily the efficacy and safety profile of omega-3 fatty acids or fibrates, both in combination with statins, and with statin alone as a control group, in local population. Lifestyle changes will also be a common parameter in all the three groups. The safety profile of combination of O3FA with statins is shown to be better than that of combination of fibrates with statins in few studies but the data is relatively scanty as far as Indian population is concerned. ${ }^{15,22-25}$ Another objective was to observe hyperuricemia in patients of dyslipidemia and the effect of lipid lowering therapies in decreasing serum uric acid levels. A study by Ogata $\mathrm{N}$ et al, investigated the effects of statins on serum uric acid (SUA) levels, retrospectively and it was found that atorvastatin reduced the SUA levels more than the other drugs. ${ }^{14}$ Another study by Uetake $\mathrm{D}$ et al, showed that administration of fenofibrate in male subjects resulted in significant reduction in SUA levels. ${ }^{13}$

\section{METHODS}

An interventional, prospective, randomized, parallel and open label study conducted in a tertiary care hospital in Punjab, India. Patients in the age group of 18-65 years of either sex with abnormal lipid profile (TGs $>150$ and $<500$ $\mathrm{mg} / \mathrm{dl}$, HDL-C $<40$ in males and $<50$ in females, LDL-C 
$>130 \mathrm{mg} / \mathrm{dl}$ ) visiting the outpatient department of a tertiary care hospital in North-West Punjab, India from October 2017 to May 2018. Patients with prior history of systemic diseases like uncontrolled hypothyroidism, pancreatitis, cholestasis, neurological dysfunction, acute liver disease or renal insufficiency, nephrotic syndrome, known case of peptic ulcer disease, also acute myocardial infarction preceding 3 months or history of muscle pain (with raised CPK levels) were excluded from the study. Patients who were pregnant or lactating mothers or those who were active alcoholic or had hypersensitivity to study drugs were also excluded from the study. Also, patients who were taking medications, known to affect plasma lipid concentration or known to interact with study medications were excluded from the study. Informed written consent was taken from all the patients enrolled after explaining study drugs, its benefits and side effects and approval from Institutional Ethics Committee was obtained before recruitment.

\section{Interventions and comparators}

The recruited patients were randomly divided into three groups (group 1, group 2 and group 3) by simple randomization using random numbers generated by computer software Research Randomizer.

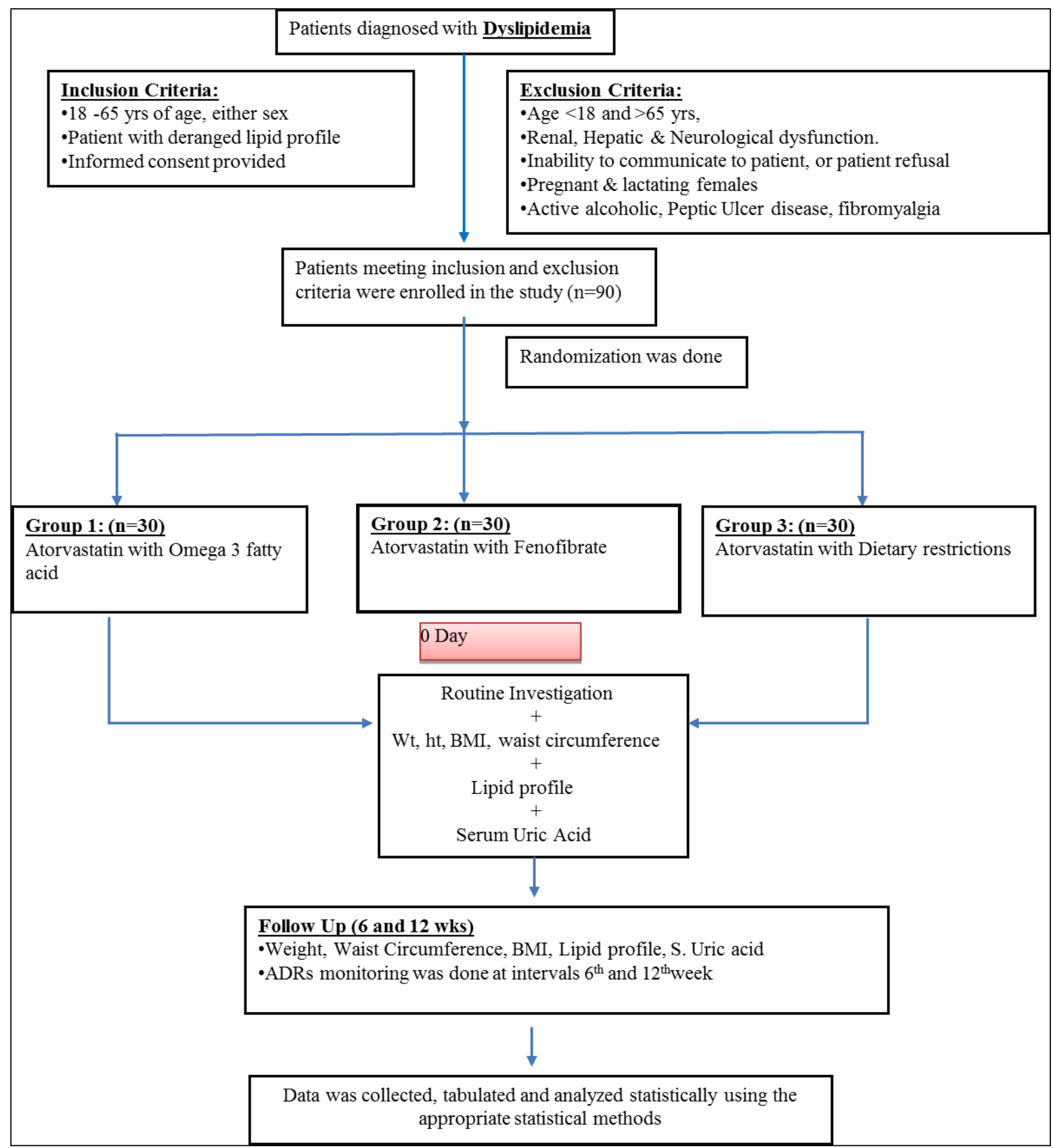

Figure 1: Consort diagram describing the flow of participants in the study. 
Group 1 received Cap Omega 3 fatty acid $2000 \mathrm{mg}$ with tab atorvastatin $20 \mathrm{mg}$ daily. Group 2 received tab fenofibrate $80 \mathrm{mg}$ with tab atorvastatin $20 \mathrm{mg}$ daily. Group 3 received only tab atorvastatin $20 \mathrm{mg}$ daily. Subjects in all the three groups were asked to follow dietary modifications as per the diet chart given to them. Drugs were administered orally on a daily basis for a total period of 12 weeks. Detailed flow chart of study protocol is described in Figure 1.

At the time of enrolment, recording of baseline vitals, general physical, systemic examination and anthropometric measurement (height, weight, waist and BMI) were done and were repeated at the end of the study. TC, HDL-C, TGs, LDL-C, SGOT and SGPT levels were done at baseline and on every follow up visit i.e. 6 weeks and 12 weeks. Routine investigations (fasting blood sugar, blood urea, serum creatinine, urine routine examination) and SUA levels were done on day 0 and after 12 weeks.

The adverse effects reported by the patients were recorded. Adverse events reported by the patients during the study period, were analysed and managed accordingly and appropriately.

Data was compiled and the statistical analysis was done using SPSS version 23. Thedata were expressed as mean \pm SD for each variable. Comparison of demographic data (age, sex) at baseline was done with the Pearson Chi square test. The baseline parameters (such as H.R., B.P., R.R., BMI, FBS, renal function test, liver function test, lipid profile and serum uric acid) of all the patients in 3 groups were analysed by One Way ANOVA. The comparison of means of percent change in Lipid profile (TCs, HDL, LDL, TGs) levels and serum uric acid levels of subjects in 3 groups at 6 and 12 weeks were analysed by one-way ANOVA. In conjunction with ANOVA, post hoc analysis using Tukey's HSD test was done for group-wise comparison of mean difference of percent change in lipid profile and serum uric acid levels at 6 and 12 weeks. The intra-group comparison of lipid profile and serum uric acid change at 12 weeks from baseline in all the 3 groups was done by paired ' $t$ ' Test. The comparison of means of percent change in other parameters (BMI, FBS, RFT, LFT) of subjects in 3 groups at 12 weeks were analysed by oneway ANOVA. The adverse drug reactions reported by the patients were tabulated and analysed by Chi square test at the end of the study. The level of significance was determined as ' $p$ ' value where $p<0.05$ was taken as statistically significant, and $\mathrm{p}<0.001$ as highly significant.

\section{RESULTS}

At baseline, demographic profile of all the patients was recorded. Mean age of 30 patients, in group 1 was $48.47 \pm 10.88$. In group 2, mean age was $51.67 \pm 9.27$ and in group 3, mean age was $51.17 \pm 9.05$. Out of total 90 patients, 47 were males and 43 were female. There was no statistically significant difference in the mean age and sex distribution amongst 3 groups. All the three groups were similar with respect to the baseline features.

There was no significant change in $\mathrm{HR}, \mathrm{BP}, \mathrm{RR}, \mathrm{BMI}$ at the end of 12 weeks amongst 3 groups. Similarly, there was no significant change in levels of fasting blood sugar, blood urea, serum creatinine, SGOT, SGPT amongst 3 groups at the end of 12 weeks.

At the end of 12 weeks, there was a fall of $24.31 \%, 33.25 \%$, $32.98 \%$ in TC, LDL-C and TG respectively, and a rise of $15.26 \%$ in HDL in Group 1 (Table 1). In group 2, there was a fall of $23.94 \%, 34.03 \%, 33.03 \%$ in TC, LDL-C and TG respectively, and a rise of $14.93 \%$ in HDL (Table 1 ).

In group 3, there was a fall of $23.88 \%, 28.15 \%, 21.31 \%$ in TC, LDL-C and TG respectively, and a rise of $12.19 \%$ in HDL (Table 1). These changes were statistically significant in each group ( $\mathrm{p}<0.001)$ (Table 2).

Table 1: Comparative mean percent change in lipid profile and SUA levels of subjects in three groups (Inter-group comparison).

\begin{tabular}{|llllll|}
\hline \multirow{2}{*}{ Parameters $(\mathrm{mg} / \mathrm{dl})$} & Group 1 & Group 2 & Group 3 & P value \\
\hline TC & \%Change at 12 weeks & $-24.31 \pm 8.91$ & $-23.94 \pm 6.88$ & $-23.88 \pm 8.73$ & 0.976 \\
\hline HDL-C & \%Change at 12 weeks & $15.26 \pm 9.61$ & $14.93 \pm 6.85$ & $12.19 \pm 8.87$ & 0.316 \\
\hline LDL-C & \%Change at 12 weeks & $-33.25 \pm 9.09$ & $-34.03 \pm 7.82$ & $-28.15 \pm 9.08$ & $0.021^{*}$ \\
\hline TGs & \%Change at 12 weeks & $-32.98 \pm 8.62$ & $-33.03 \pm 6.87$ & $-21.31 \pm 9.85$ & $<0.001^{* * *}$ \\
\hline SUA & \%Change at 12 weeks & $-5.74 \pm 6.78$ & $-9.44 \pm 7.06$ & $-4.85 \pm 5.07$ & $0.015^{*}$ \\
\hline
\end{tabular}

$* \mathrm{p}<0.05$-significant, ${ }^{* *} \mathrm{p}<0.01$ - very significant; $* * * \mathrm{p}<0.001$ highly significant, (TC-Total Cholesterol, HDL-C - High Density Lipoprotein, LDL-C - Low Density Lipoprotein, TGs- Triglycerides and SUA-Serum Uric Acid).

Percent TC reduction and HDL-C rise was more in group 1 compared to group 2 followed by group 3, $(\mathrm{Gp} 1>\mathrm{Gp} 2>\mathrm{Gp} 3)$, whereas LDL-C reduction and TG reduction was more in group 2 compared to group 1 followed by group 3, (Gp2>Gp1>Gp3) (Table 1). Both group 1 and 2 showed highly significant fall in TG levels 
( $\mathrm{p}<0.001$ ) in comparison to group 3 whereas comparative reduction in TG levels between groups 1 and 2 was not significant (Table 3). Group 2 also showed significant fall in LDL-C levels ( $\mathrm{p}<0.01$ ) in comparison to group 3 (Table $3)$.

Table 2: Mean change from baseline levels in lipid profile and serum uric acid within study groups.

\begin{tabular}{|c|c|c|c|c|}
\hline Parameters (mg/dl) & O day (Mean \pm SD) & 12 weeks $($ Mean \pm SD) & Mean change & P-value \\
\hline \multicolumn{5}{|l|}{ Group 1} \\
\hline $\mathrm{TC}$ & $242.60 \pm 30.70$ & $182.03 \pm 18.53$ & -60.57 & $\mathrm{P}<0.001$ \\
\hline HDL-C & $36.50 \pm 05.99$ & $41.70 \pm 05.40$ & 05.20 & $\mathrm{P}<0.001$ \\
\hline LDL-C & $163.33 \pm 26.83$ & $107.67 \pm 16.28$ & -55.66 & $\mathrm{P}<0.001$ \\
\hline TGs & $238.47 \pm 51.95$ & $157.33 \pm 27.14$ & -81.13 & $\mathrm{P}<0.001$ \\
\hline SUA & $5.85 \pm 01.44$ & $5.47 \pm 01.22$ & -0.378 & $\mathrm{P}<0.001$ \\
\hline \multicolumn{5}{|l|}{ Group 2} \\
\hline $\mathrm{TC}$ & $228.67 \pm 20.35$ & $173.47 \pm 17.77$ & -55.20 & $\mathrm{P}<0.001$ \\
\hline HDL-C & $36.90 \pm 04.51$ & $42.30 \pm 04.78$ & 05.40 & $\mathrm{P}<0.001$ \\
\hline LDL-C & $158.57 \pm 23.77$ & $104.00 \pm 17.02$ & -54.57 & $\mathrm{P}<0.001$ \\
\hline TGs & $237.57 \pm 55.49$ & $156.70 \pm 27.21$ & -80.87 & $\mathrm{P}<0.001$ \\
\hline SUA & $6.231 \pm 01.43$ & $05.59 \pm 01.41$ & -00.64 & $\mathrm{P}<0.001$ \\
\hline \multicolumn{5}{|l|}{ Group 3} \\
\hline $\mathrm{TC}$ & $240.20 \pm 38.76$ & $180.43 \pm 20.256$ & -59.76 & $\mathrm{P}<0.001$ \\
\hline HDL-C & $38.25 \pm 05.63$ & $42.67 \pm 05.09$ & 04.41 & $\mathrm{P}<0.001$ \\
\hline LDL-C & $161.57 \pm 27.08$ & $114.73 \pm 16.35$ & -46.83 & $\mathrm{P}<0.001$ \\
\hline TGs & $240.33 \pm 50.087$ & $188.07 \pm 42.637$ & -52.27 & $\mathrm{P}<0.001$ \\
\hline SUA & $5.77 \pm 01.63$ & $5.46 \pm 01.50$ & -00.31 & $\mathrm{P}<0.001$ \\
\hline
\end{tabular}

$\mathrm{p}<0.001$ highly significant, (TC-Total Cholesterol, HDL-C - High Density Lipoprotein, LDL-C - Low Density Lipoprotein, TGs- Triglycerides and SUASerum Uric Acid).

There was a fall of $5.74 \%, 9.44 \%$ and $4.85 \%$ in SUA levels in group 1, group 2 and group 3 respectively (Table 1).
These changes were statistically significant in each group $(\mathrm{p}<0.001)$ (Table 2).

Table 3: Group-wise comparison of mean reduction in lipid profile and SUA levels at 12 weeks.

\begin{tabular}{|lllllll|}
\hline $\begin{array}{l}\text { Parameters } \\
(\mathbf{m g} / \mathbf{d l})\end{array}$ & $\begin{array}{l}\text { Group 1 vs group 2 } \\
\begin{array}{l}\text { Difference of mean } \\
\text { reduction }\end{array}\end{array}$ & $\mathbf{P}$ & $\begin{array}{l}\text { Group 1 vs group 3 } \\
\text { Difference of } \\
\text { mean reduction }\end{array}$ & $\mathbf{P}$ & \multicolumn{3}{c|}{$\begin{array}{l}\text { Group 2 vs group 3 } \\
\text { Difference of } \\
\text { mean reduction }\end{array}$} & $\begin{array}{l}\text { P } \\
\text { TC }\end{array}$ & 5.37 & 0.983 & 0.81 & 0.977 & 4.56 & 1.00 \\
\hline HDL-C & 0.20 & 0.990 & 0.79 & 0.350 & 0.99 & 0.431 \\
\hline LDL-C & 1.09 & 0.935 & 8.83 & 0.065 & 7.74 & $0.027 *$ \\
\hline TGs & 0.26 & 1.00 & 29.06 & $<0.001 * * *$ & 28.6 & $<0.001 * * *$ \\
\hline SUA & 0.262 & 0.068 & 0.068 & 0.851 & 0.33 & $0.017 *$ \\
\hline
\end{tabular}

*p $<0.05$ - significant, **p <0.01- very significant; ***p <0.001 highly significant, (TC-Total Cholesterol; HDL-C - High Density Lipoprotein, LDL-C Low Density Lipoprote in TGs- Triglycerides and SUA-Serum Uric Acid).

Table 4: Adverse effects shown by three groups in study period.

\begin{tabular}{|lllll|}
\hline Adverse effects & Group 1 & Group 2 & Group 3 & P value \\
\hline Gastrointestinal upset (Dyspepsia, nausea or vomiting) & 3 & 4 & 4 & 0.914 \\
\hline Myalgia (mild muscle aches and tiredness) & 0 & 2 & 0 & 0.129 \\
\hline Increase in SGOT/SGPT (>3 times normal) & 0 & 0 & 0 & - \\
\hline Increase in creatine phosphokinase (CPK >5 times normal) & 0 & 0 & 0 & - \\
\hline Hypersensitivity (mild itching) & 2 & 0 & 0 & 0.129 \\
\hline
\end{tabular}

$\mathrm{p}<0.05$ - significant. 
Percentage change in serum uric acid levels at the end of 12 weeks was more in group 2 compared to group 1 followed by group 3, (Gp2>Gp1>Gp3) (Table 1).

Group 2 showed significant fall in SUA levels in comparison to group 3 ( $p<0.01$ ), whereas fall in other two intergroup comparisons (group 1 vs group 2, group 1 vs group 3) was not significant (Table 3 ).

Most common adverse effect in all the 3 groups was gastrointestinal disturbances in the form of dyspepsia, nausea, vomiting, etc., and its incidence was comparable amongst 3 groups (10-13.33\%). There were $2(6.67 \%)$ cases of mild itching and $2(6.67 \%)$ cases of mild muscle aches and tiredness in Group 1 and 2 respectively which showed no statistical significant difference amongst the groups (Table 4).

\section{DISCUSSION}

Trials of atorvastatin combined either with fenofibrate or with O3FA have shown promising results in lowering serum triglyceride levels and increasing HDL-C levels but there are very few studies where both triglyceride lowering agents have been compared with each other. This study was designed to compare the efficacy and safety of O3FA and fenofibrate with background atorvastatin therapy and dietary restrictions in a total of 90 patients of atherogenic dyslipidemia. Clinical efficacy parameters were measured at 6 and 12 week from baseline. Adverse events reported during treatment period were tabulated and analysed for all the 3 groups.

The study groups were randomly allocated and there was no significant difference in mean age $(p>0.05)$, sex distribution ( $p>0.05)$ and baseline biochemical parameters and BMI mean values $(p>0.05)$ of the subjects in three groups.

All three groups registered a significant mean percent change in serum TC after 12 weeks of treatment, which was $24.31 \%, 23.94 \%$ and $23.88 \%$ in group 1,2 and 3 respectively (Table 1). The fall in TC was statistically significant in each group ( $\mathrm{p}<0.001)$ (Table 2). However, when the groups were compared with each other, no statistically significant difference was observed (Table 1). It could be attributed to background atorvastatin therapy in all the three groups. ${ }^{26}$ Combination of O3FA with atorvastatin achieved relatively greater reduction in total cholesterol compared to fenofibrate and atorvastatin combination, which is not in concordance with other works like Caniato RN et al, and Abourbih S et al. ${ }^{27,28}$

The mean percent change in serum HDL-C after treatment at 12 weeks was $15.26 \%, 14.93 \%$ and $12.19 \%$ in group 1, 2 and 3 respectively (Table 1). There was a significant increase in HDL-C levels from the baseline values in all three groups ( $p$ <0.001) (Table 2). However, when the groups were compared with each other, no statistically significant difference was observed (Table 1). Similar observations had been reported in study by Agourdis AP et al. $^{29}$

The mean percent change in serum LDL-C from baseline after treatment at 12 weeks was $33.25 \%, 34.03 \%$ and $28.15 \%$ in group 1, 2 and 3 respectively (Table 1). The fall in LDL-C was statistically significant in each group (p $<0.001$ ) (Table 2) and when the groups were compared with each other, statistically significant difference was observed ( $\mathrm{p}<0.05$ ) (Table 1). Fenofibrate in combination with atorvastatin (group 2), achieved a greater and significant reduction in LDL-C, as compared to atorvastatin alone (group 3) $(\mathrm{p}<0.05)$, whereas other two inter-group comparisons were not significant (Table 3). This is in agreement with a study by Agouridis AP et al. ${ }^{29}$

A similar trend was observed in case of serum TG levels. The mean percent change in serum TG after treatment at 12 weeks was $32.98 \%, 33.03 \%, 21.31 \%$ in group 1, 2 and 3 respectively (Table 1). The fall in TG was statistically significant in each group ( $\mathrm{p}<0.001)$ (Table 2) and when the groups were compared with each other, statistically significant difference was observed ( $\mathrm{p}<0.001)$ (Table 1). Between group 1 and 3 as well as group 2 and 3 , a significant difference in TG reduction was observed ( $p$ $<0.001$ ), but there was no significant difference as regards TG reduction in group 1 and 2 (Table 3). This is in conformity with other similar studies. ${ }^{29-31}$

The mean percent change in serum uric acid after the treatment at 12 weeks was $5.74 \%, 9.44 \%$ and $4.85 \%$ in groups 1,2 and 3 respectively ( $\mathrm{p}<0.001)$ (Table 1). Combination of atorvastatin and fenofibrate showed greater percentage reduction in SUA levels compared to O3FA and atorvastatin or atorvastatin alone (Table 3). Earlier studies have shown SUA levels lowering effects of both fenofibrate or atorvastatin used alone. ${ }^{14,32}$ Present study reinforces this fact. Though it is not expected that reduction of uric acid can alter atherogenesis, some patients with hyperuricemia receiving lipid-lowering treatment may benefit from this peculiar effect. ${ }^{32}$

The parameters such as BMI, FBS, blood urea, serum creatinine, SGOT, SGPT showed no statistically significant change at 12 weeks from baseline, amongst 3 groups.

The most common side effect observed in group 1 (atorvastatin+omega 3 fatty acids) was gastrointestinal disturbances in the form of dyspepsia, loose stools or nausea (10\%), followed by mild itching $(6.67 \%)$. Backes $\mathrm{J}$ et al, and Chang $\mathrm{CH}$ et al, showed similar side effects in their respective studies. ${ }^{33,34}$ With group 2 (atorvastatin+ fenofibrate) the most common side effects observed was gastrointestinal disturbances in the form of dyspepsia, loose stools or nausea $(13.33 \%)$ patients, followed by mild muscle aches and tiredness $(6.67 \%)$. Similar side effects were observed in the study conducted by Nicola et al, on combination of statin with fenofibrate. ${ }^{35}$ Fenofibrate is documented to be relatively safer for combined use with 
statins, as compared with other fibrates like gemfibrozil which increase the risk of myopathy. ${ }^{36}$ Most common side effects observed in group 3 (atorvastatin+dietary restrictions) was gastrointestinal disturbances in 4 (13.3\%) patients. No other documented adverse effects were reported. ${ }^{37,38}$ Comparing the incidence of these adverse effects seen in three groups, no statistically significant difference amongst them was seen $(p>0.05)$. No patient had significant rise in serum aminotransferases $(>3$ times normal) levels and no patient opted out of the study due to any serious side effects (Table 4).

\section{CONCLUSION}

No statistically significant difference was observed between omega-3 fatty acids and fenofibrate (both with background atorvastatin therapy) as regards their efficacy in lowering serum TG and LDL-C levels in patients of atherogenic dyslipidemia. A secondary objective of this study was to determine relative effects on SUA levels and combination of fenofibrate and atorvastatin showed better outcome compared to other groups. With respect to the safety, drugs in all the three groups were comparably well tolerated. Omega-3 fatty acids, therefore, seem to be a good alternative to fibrates in patients not tolerating the latter.

\section{Funding: No funding sources}

Conflict of interest: None declared

Ethical approval: The study was approved by the Institutional Ethics Committee

\section{REFERENCES}

1. Chandra SK, Bansal M, Nair T, Iyengar SS, Gupta R, Manchanda SC, et al. Consensus statement on management of dyslipidemia in Indian subjects. Ind Heart J. 2014;66(3):S1-51.

2. Mulukutla SR, Venkitachalam L, Marroquin OC, Kip KE, Aiyer A, Edmundowicz D, et al. Population variations in atherogenic dyslipidemia: a report from the Heart SCORE and India SCORE Studies. J Clin Lipidol. 2008;2(6):410-7.

3. Sharma RK, Singh VN, Reddy HK. Thinking beyond low-density lipoprotein cholesterol: strategies to further reduce cardiovascular risk. Vasc Health Risk Manag. 2009;5:793-9.

4. Ramachandran A, Snehalatha C, Satyavani K, Sivasankari S, Vijay V. Metabolic syndrome in urban Asian Indian adults: a population study using modified ATP III criteria. Diab Res Clin Pract. 2003;60(3):199204.

5. Mohan V, Shanthirani S, Deepa R, Premalatha G, Sastry NG, Saroja R, et al. Intra-urban differences in the prevalence of the metabolic syndrome in southern India: the Chennai Urban Population Study (CUPS No. 4). Diab Med J Brit Diab Assoc. 2001;18(4):2807.

6. Austin MA, King MC, Vranizan KM, Krauss RM. Atherogenic lipoprotein phenotype. a proposed genetic marker for coronary heart disease risk. Circulation. 1990;82(2):495-506.

7. Vinik AI. The metabolic basis of atherogenic dyslipidemia. Clin Cornerstone. 2005;7(2-3):27-35.

8. Musunuru K. Atherogenic dyslipidemia: cardiovascular risk and dietary intervention. Lipids. 2010;45(10):907-14.

9. Grundy SM. Hypertriglyceridemia, atherogenic dyslipidemia, and the metabolic syndrome. Am J Cardiol. 1998;81(4A):18B-25B.

10. Lehto S, Niskanen L, Rönnemaa T, Laakso M. Serum uric acid is a strong predictor of stroke in patients with non-insulin-dependent diabetes mellitus. Stroke. 1998;29(3):635-9.

11. Bickel C, Rupprecht HJ, Blankenberg S, Rippin G, Hafner G, Daunhauer A, et al. Serum uric acid as an independent predictor of mortality in patients with angiographically proven coronary artery disease. Am J Cardiol. 2002;89(1):12-7.

12. Choi HK, Ford ES. Prevalence of the metabolic syndrome in individuals with hyperuricemia. Am J Med. 2007;120(5):442-7.

13. Uetake D, Ohno I, Ichida K, Yamaguchi Y, Saikawa $\mathrm{H}$, Endou $\mathrm{H}$, et al. Effect of fenofibrate on uric acid metabolism and urate transporter 1. Intern Med Tokyo Jpn. 2010;49(2):89-94.

14. Ogata N, Fujimori S, Oka Y, Kaneko K. Effects of three strong statins (atorvastatin, pitavastatin, and rosuvastatin) on serum uric acid levels in dyslipidemic patients. Nucleosides Nucleotides Nucleic Acids. 2010;29(4-6):321-4.

15. Manjunath CN, Rawal JR, Irani PM, Madhu K. Atherogenic dyslipidemia. Indian J Endocrinol Metab. 2013;17(6):969-76.

16. Taskinen M-R. Diabetic dyslipidaemia: from basic research to clinical practice. Diabetol. 2003;46(6):733-49.

17. Adiels M, Olofsson S-O, Taskinen M-R, Borén J. Overproduction of very low-density lipoproteins is the hallmark of the dyslipidemia in the metabolic syndrome. Arterioscler Thromb Vasc Biol. 2008;28(7):1225-36.

18. Packard C, Caslake M, Shepherd J. The role of small, dense low-density lipoprotein (LDL): a new look. Int J Cardiol. 2000;74:S17-22.

19. Witztum JL, Steinberg D. Role of oxidized lowdensity lipoprotein in atherogenesis. J Clin Invest. 1991;88(6):1785-92.

20. Witztum JL, Steinberg D. The oxidative modification hypothesis of atherosclerosis: does it hold for humans? Trends Cardiovasc Med. 2001;11(3-4):93-102.

21. Gurgle HE, Blumenthal DK. Drug Therapy for Dyslipidemias. In: Brunton LB, Dandan RH, Knollman BC, eds. Goodman and Gilman's The Pharmacological Basis of Therapeutics 12th ed. New York, NY: Mc Graw-Hill; 2018:605-18.

22. Noce MA, Bertucci P, Villahermosa MS, Zenobi R, Castagnola V. Atherosclerosis, dyslipidemia, and inflammation: the significant role of polyunsaturated fatty acids. Int Sch Res Not. 2013;2013:e191823. 
23. Barter P, Ginsberg HN. Effectiveness of combined statin plus omega-3 fatty acid therapy for mixed dyslipidemia. Am J Cardiol. 2008;102(8):1040-5.

24. Yokoyama M, Origasa H, Matsuzaki M, Matsuzawa Y, Saito Y, Ishikawa Y, et al. Effects of eicosapentaenoic acid on major coronary events in hypercholesterolaemic patients (JELIS): a randomised open-label, blinded endpoint analysis. Lancet Lond Engl. 2007;369(9567):1090-8.

25. Durrington PN, Bhatnagar D, Mackness MI, Morgan J, Julier K, Khan MA, et al. An omega-3 polyunsaturated fatty acid concentrate administered for one-year decreased triglycerides in simvastatin treated patients with coronary heart disease and persisting hypertriglyceridaemia. Heart $\mathrm{Br}$ Card Soc. 2001;85(5):544-8.

26. Shuhaili MF, Samsudin IN, Stanslas J, Hasan S, Thambiah SC. Effects of different types of statins on lipid profile: a perspective on Asians. Int J Endocrinol Metab. 2017;15(2)

27. Abourbih S, Filion KB, Joseph L, Schiffrin EL, Rinfret S, Poirier P, et al. Effect of fibrates on lipid profiles and cardiovascular outcomes: a systematic review. Am J Med. 2009;122(10):962.e1-8.

28. Caniato RN, Alvarenga ME, Garcia-Alcaraz MA. Effect of omega-3 fatty acids on the lipid profile of patients taking clozapine. Aust N Z J Psychiatry. 2006;40(8):691-7.

29. Agouridis AP, Kostapanos MS, Tsimihodimos V, Kostara C, Mikhailidis DP, Bairaktari ET, et al. Effect of rosuvastatin monotherapy or in combination with fenofibrate or $\omega-3$ fatty acids on lipoprotein subfraction profile in patients with mixed dyslipidaemia and metabolic syndrome. Int $\mathrm{J}$ Clin Pract. 2012;66(9):843-53.

30. Jones PH, Cusi K, Davidson MH, Kelly MT, Setze $\mathrm{CM}$, Thakker K, et al. Efficacy and safety of fenofibric acid co-administered with low- or moderate-dose statin in patients with mixed dyslipidemia and type 2 diabetes mellitus: results of a pooled subgroup analysis from three randomized, controlled, double- blind trials. Am J Cardiovasc Drugs Drugs Devices Interv. 2010;10(2):73-84.

31. Bradberry JC, Hilleman DE. Overview of omega-3 fatty acid therapies. Pharm Ther. 2013;38(11):681-91.

32. Serna DL. Fenofibrate decreases plasma fibrinogen, improves lipid profile, and reduces uricemia. Clin Pharmacol. 1999;66(2):7.

33. Backes J, Anzalone D, Hilleman D, Catini J. The clinical relevance of omega-3 fatty acids in the management of hypertriglyceridemia. Lipids Heal Dis. 2016;15(1):118.

34. Chang C-H, Tseng P-T, Chen N-Y, Lin P-C, Lin P-Y, Chang JP-C, et al. Safety and tolerability of prescription omega-3 fatty acids: A systematic review and meta-analysis of randomized controlled trials. Prostaglandins Leukot Essent Fatty Acids. 2018;129:1-12.

35. Tarantino N, Santoro F, De Gennaro L, Correale M, Guastafierro F, Gaglione A, et al. Fenofibrate/simvastatin fixed-dose combination in the treatment of mixed dyslipidemia: safety, efficacy, and place in therapy. Vasc Health Risk Manag. 2017;13:29-41.

36. Whitfield LR, Porcari AR, Alvey C, Abel R, Bullen W, Hartman D. Effect of gemfibrozil and fenofibrate on the pharmacokinetics of atorvastatin. J Clin Pharmacol. 2011;51(3):378-88.

37. Li H, Wang C, Zhang S, Sun S, Li R, Zou M, et al. Safety Profile of Atorvastatin $80 \mathrm{mg}$ : A Meta-Analysis of 17 Randomized Controlled Trials in 21,910 Participants. Drug Saf. 2016;39(5):409-19.

38. Chan JCN, Kong APS, Bao W, Fayyad R, Laskey R. Safety of atorvastatin in Asian patients within clinical trials. Cardiovasc Ther. 2016;34(6):431-40.

Cite this article as: Arora A, Singh NR, Sharma R. Comparative study of efficacy and safety of omega 3 fatty acids and fenofibrate with background atorvastatin therapy in patients of atherogenic dyslipidaemia. Int J Basic Clin Pharmacol 2019;8:1145-52. 